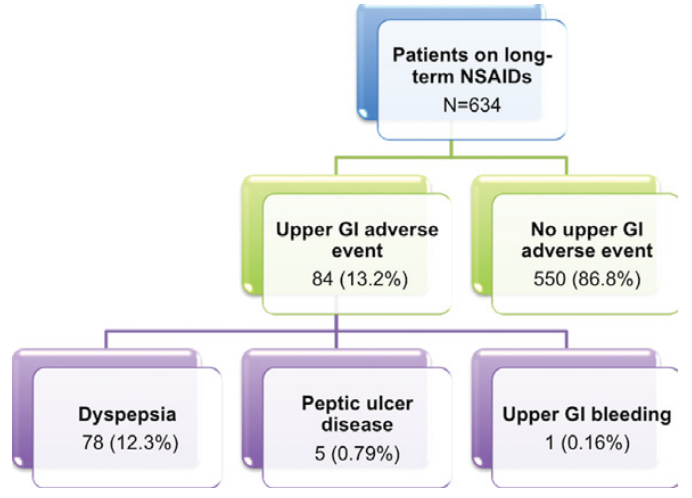

tology patients suggests judicious use of NSAIDs in tertiary care setting resulting in a low cost implication for the management of these events.

Disclosure of Interest: None declared

DOI: 10.1136/annrheumdis-2017-eular.3523

\section{THU0639 RHEUMATOLOGICAL MEDICATION USE IN PREGNANCY, PLANNING PREGNANCY, AND BREASTFEEDING AT MOTHERSAFE DURING THE YEARS 2010 TO 2015}

S.-N. Luong ${ }^{1}$, D. Kennedy ${ }^{2}$, J.V. Bertouch ${ }^{1} .{ }^{1}$ Rheumatology, Prince of Wales Hospital; ${ }^{2}$ Clinical Genetics, Royal Hospital for Women, Randwick, Australia

Background: MotherSafe is a free, statewide, phone based counselling service for the general public and healthcare professionals concerned about exposures during pregnancy, pregnancy planning and breastfeeding (1). Obstetric drug information services such as MotherSafe are important in guiding decisionmaking in pregnancy, breastfeeding and planning of future pregnancies, and are increasingly used worldwide by both patients and healthcare providers (1, 2). At MotherSafe, phone advice is provided by trained telephone counsellors who are generally pharmacists. Data from each phone call is entered onto an electronic document. Some patients are then referred onto the MotherSafe clinic for specialised counselling by a clinical geneticist.

Objectives: To analyse data including patient and medication characteristics and trends from phone calls made to MotherSafe regarding disease modifying antirheumatic drugs (DMARDs) and biologic DMARDs (bDMARDs) from January 2010 to December 2015 inclusive.

Methods: Retrospective study of phone calls made to MotherSafe from January 2010 to December 2015 regarding conventional DMARDs and biologic DMARDs. SPSS software facilitated statistical analysis.

Results: A total of 135,115 phone calls were made to MotherSafe from 2010-2015 with $2611(1.93 \%)$ phone calls pertaining to DMARDs and bDMARDs.

Of these 2611 phone calls, $65.4 \%$ were made by patients and $13.5 \%$ by general practitioners. Most phone calls were made in metropolitan New South Wales $(69.3 \%)$. $43 \%$ of phone calls were concerning exposures during breastfeeding, followed by exposures during pregnancy $(32.9 \%)$, exposures whilst planning pregnancy (17.7\%) and paternal exposures (2.9\%). Where a specific diagnosis was given, inflammatory bowel disease was the most common indication (18.4\%), followed by rheumatoid arthritis $(8.2 \%)$. Corticosteroids were the most common medication exposure (37.3\%), followed by azathioprine (18.8\%), sulfasalazine $(11.2 \%)$ and methotrexate $(8.5 \%)$.

Most callers just received phone advice, especially if the call was just regarding breastfeeding exposures (73.4\%). 383 callers (14.7\%) were referred onto the MotherSafe clinic, which is run by a clinical geneticist.

bDMARDs made up $9.5 \%$ of calls with calls tending to increase over the years, but there was a slight decrease in 2012 and 2013 albeit with small numbers. TNF inhibitors still made up the majority of calls regarding bDMARDs.

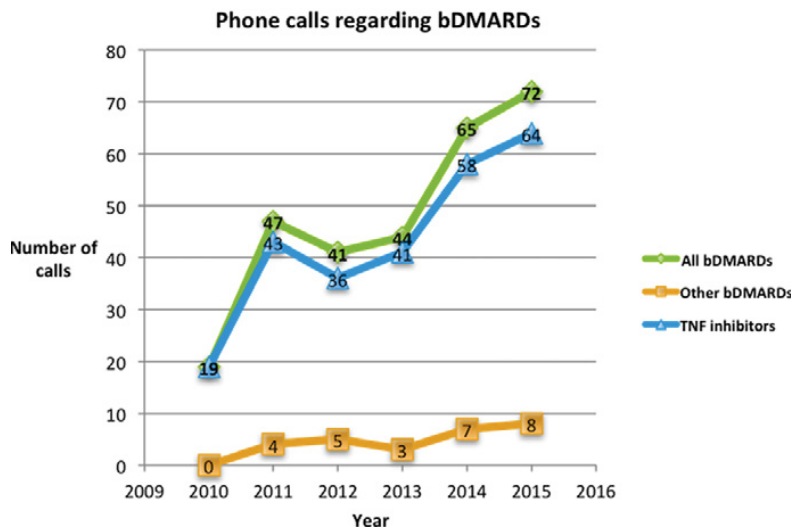

Conclusions: This study evaluated the only obstetric medicine exposure information service in New South Wales, Australia. It is the first time that DMARDs and bDMARDs have been analysed for MotherSafe. There was a trend to increasing number of calls regarding bDMARDs over 2010 to 2015, which presumably reflects change in prescribing patterns. This study highlights the need for services like MotherSafe so patients and health care professionals can receive evidence based information and make choices about treatment in pregnancy.

References:

[1] Kennedy D, Eamus M, Hill M, Oei JL. Review of calls to an australian teratogen information service regarding psychotropic medications over a 12 year period. Aust N Z J Obstet Gynaecol. 2013;53(6):544-52. doi: 10.1111/ajo.12129.

[2] De Santis $M$ et al. Prenatal drug exposure and teratological risk one year experience of an italian teratology information service. Med Sci Monit 20-8;14:PH1-8.

Acknowledgements: MotherSafe staff

Disclosure of Interest: None declared

DOI: 10.1136/annrheumdis-2017-eular.5560

\section{THU0640 SECOND LINE TREATMENT PERSISTENCE AND COSTS AMONG PATIENTS WITH IMMUNE-MEDIATED RHEUMATIC DISEASES TREATED WITH SUBCUTANEOUS TNF-ALPHA INHIBITORS}

J. Dalén ${ }^{1}$, A. Svedbom ${ }^{1}$, C.M. Black ${ }^{2}$, S. Kachroo ${ }^{2}$. ${ }^{1}$ Mapi group, Stockholm, Sweden; ${ }^{2}$ Merck \& Co., Inc., Kenilworth, United States

Background: For some patients with Immune Mediated Rheumatic Disease (IMRD) discontinuing 1st line treatment with a subcutaneous Tumor Necrosis Factor-alpha inhibitor (SC-TNFi), 2nd line treatment with another SC-TNFi may be appropriate.

Objectives: The primary objective of this study was to describe treatment persistence with 2nd line SC-TNFi stratified by agent in patients with IMRD in Sweden. The secondary objective was to explore the impact of non-persistence with a second SC-TNFi on health care costs.

Methods: We conducted a retrospective study on treatment persistence and health care costs using data from health registers. Adults ( $\geq 18$ years old) previously treated with one SC-TNFi and subsequently prescribed a second SC-TNFi were identified through prescriptions for adalimumab (ADA), etanercept (ETA), certolizumab pegol (CZP) and golimumab (GLM) between 5/6/2010 and $12 / 31 / 2012$. Prescriber specialty and department were used to exclude patients treated for diseases other than IMRD. Persistence up to 3 years was estimated using non-parametric survival analysis. Given differences in baseline characteristics, analyses were conducted on propensity score matched (PSM) cohorts. Matching was based on age, gender, index year, diagnosis, Charlson Comorbidity Index and non-biologic DMARD use. Non-treatment health care costs were captured 12 months pre and post initiation of 2nd line SC-TNFi treatment and stratified by persistence status at 6 months.

Results: In total, 845 patients were identified (ADA: 316, ETA: 202, CZP: 140, GLM: 187). PSM cohorts were generated as GLM vs ADA, GLM vs ETA and GLM vs CZP, with 187, 164, and 113 matched pairs, respectively. GLM exhibited statistically significant higher persistence than ADA over 3 years (Figure; $p=0.040$ ) and numerically, but not statistically significant, higher persistence than ETA and CZP at 12 and 24 months. Persistent and non-persistent patients had similar mean total cost 12 months pretreatment initiation (USD 5,185 vs USD 5,064, $\mathrm{p}=0.750$ ). During the 12 months post treatment initiation, persistent patients had lower mean total costs (USD 4,377 vs USD 6,605), corresponding to a difference in difference of USD $2,228(\mathrm{p}<0.001)$.

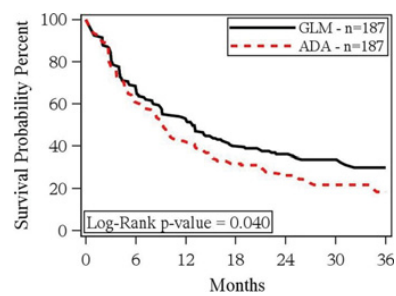

Conclusions: In patients previously treated with a SC-TNFi, GLM exhibited significantly better persistence than ADA and numerically higher persistence than ETA and CZP at 12 and 24 months, findings that are similar to results observed in 1 st line SC-TNFi patients ${ }^{1}$. Given the lower healthcare costs for persistent patients, the choice of 2nd line SC-TNFi among eligible patients may merit careful consideration given its impact on patients and payers.

References:

[1] Dalén, J, et al. Treatment persistence among patients with immune-mediated rheumatic disease newly treated with subcutaneous TNF-alpha inhibitors and costs associated with non-persistence. Rheumatol International (2016): 1-9.

Disclosure of Interest: J. Dalén Consultant for: Merck \& Co., Inc., Employee of: Mapi group, A. Svedbom Consultant for: Merck \& Co., Inc., Employee of: Mapi group, C. Black Shareholder of: Merck \& Co., Inc., Employee of: Merck \& Co., Inc., S. Kachroo Shareholder of: Merck \& Co., Inc., Employee of: Merck \& Co., Inc. DOI: 10.1136/annrheumdis-2017-eular.2654 